Bangladesh J Med Microbiol 2019; 13 (1): 1-3

Bangladesh Society of Medical Microbiologists

\title{
Editorial
}

\section{Acinetobacter baumannii Hospital Acquired Infections.}

\author{
Md. Ruhul Amin Miah
}

Over the last decades, Acinetobacter baumannii has globally emerged as a highly troublesome nosocomial pathogen, mainly in patients in intensive care units (ICUs). It is most important nosocomial pathogens because of two important characteristics: first, its ability to survive in the hospital environment on animate and inanimate surfaces for long periods with the risk of prolonged endemic infections and second, its resistance to multiple antibiotics including carbapenems- which complicates treatment and to colonize susceptible patients treated with broad-spectrum antibiotic. ${ }^{1,2}$ Multidrug-resistant (MDR) A. baumannii strains are associated with infections such as ventilator-associated pneumonia(VAP), septicemia, urinary tract infection, soft skin infection, wound infection, and meningitis, especially in immunocompromised patients in ICU settings. ${ }^{3}$ The success of A. baumannii as an emerging nosocomial pathogen is mostly due to its efficiency in acquiring new antibiotic-resistant determinants. ${ }^{4}$ which may have contributed to the high antibiotic index as classified by the World Health Organization (WHO). The carbapenem-resistant A. baumannii is now grouped among the leading causes of bacterial nosocomial infections throughout the world. This special category of pathogens termed as "ESKAPE" consists of Enterococcus faecium, Staphylococcus aureus, Klebsiella pneumoniae, Acinetobacter baumannii, Pseudomonas aeruginosa, and Enterobacter spp. ${ }^{5}$

Clinical significance has been largely driven by a remarkable ability to acquire or upregulate various resistance determinants, making it one of the most successful multidrug-resistant (MDR) organisms threatening current antibiotic therapy. ${ }^{6}$ A. baumannii is endowed with multiple mechanisms of survival under a wide range of environments, potentiating capacity for hospital spread. ${ }^{7}$ Among the attributable mortalities in patients with A. baumannii healthcare-associated infections, ventilatorassociated pneumonia and bloodstream infections are the most common in the intensive care unit (ICU) ${ }^{8}$ with increasing reports of community-acquired A. baumannii infections. ${ }^{9}$ Extensively drug-resistant (XDR) and

Prof. Md. Ruhul Amin Miah

Department of Microbiology and Immunology

Bangabandhu Sheikh Mujib Medical University

E-mail: ruhulamin53@gmail.com pandrug-resistant (PDR) isolates of A. baumannii is also accumulating in different countries. ${ }^{10}$

Treating carbapenem-resistant A. baumannii infections is very challenging since they are naturally resistant to antibiotics and therefore associated with poor clinical outcomes. These infections are generally resistant to three or more groups of antibiotic families from quinolones, cephalosporins, $\beta$-lactams, aminoglycosides to carbapenems. ${ }^{11}$ Due to its ability to accept exogenous genetic material and overexpress endogenous resistance genes, it has quickly resulted in the appearance of the MDR phenotype within multiple clonal lineages. In addition to antibiotic resistance, the ability to adhere and produce biofilm on both biotic and abiotic surfaces has been shown to be a virulence factor in many clinical isolates. $^{12}$

The World Health Organization lists the carbapenem resistant A. baumannii on the pathogen critical priority list ${ }^{13}$, making it an increasingly growing public health problem worldwide. Multidrug (MDR) resistance among A. baumannii strains is driven by rapid gene mutations or transfer of exogenous resistance genes by mobile genetic elements, such as plasmids, transposons or insertion sequences. Resistance to beta-lactam and aminoglycosides is also a rapidly growing public health problem globally. Circulation of carbapenem-resistant A. baumannii strains aggravates the antibiotic resistance scenery, making patient's management challenging. ${ }^{14}$ As a result, occurrence of transmission networks associated with MDR in clinical settings has become a growing health issue in different regions of the world. ${ }^{15}$

Antibiotic resistance in A. baumannii is mediated by enzymatic degradation of antibiotics, mutations/modification of target sites, reduced expression of porins, and overexpression of multidrug efflux pumps. ${ }^{16,17}$ However, resistance to carbapenem is often mediated by $\beta$-lactamases including carbapenem-hydrolyzing class $\mathrm{D}$ $\beta$-lactamases (CHDLs) and metallo- $\beta$-lactamases (MBLs). Resistance by class D $\beta$-lactamases (CHDLs), also known as oxacillinases, is mainly mediated by the production of carbapenemase enzymes encoded by genes of the blaoXA-23, blaoXA-40, and blaoXA-58-like lineage; however, blaoXA-23 is reported to be the most prevalent 
worldwide. ${ }^{18,19}$ Transposable elements such as insertion sequences (ISAbal) have an important role in carbapenem resistance in A. baumannii and are present upstream at promoter regions of the blaoXA-23, blaoXA-40, blaoXA-58, and blaoxA-5I genes causing overexpression of these resistant genes. ${ }^{20}$

In Bangladesh 96\% A. baumannii was detected as MDR. The antimicrobial resistance genes such as blaampC (both chromosomal and plasmid mediated) and carbapenemase genes (blaMBLs and blaKPC) were observed among $95.83 \%$ cephalosporin resistant A. baumannii. ${ }^{21}$

According to the Centers for Disease Control and Prevention (CDC) in 2013, (63\%) out of 12,000 annual infections in the USA were due to MDR carbapenemresistant A. baumannii, leading to about 500 deaths annually. ${ }^{22}$ By the late 1990s, carbapenems were the most important antimicrobial drugs of choice by clinicians as they represent the "last-line" drugs for the treatment of infections caused by MDR A. baumannii due to their high efficiency and low toxicity. ${ }^{23}$

The growing rate of carbapenem resistant A. baumannii which continues to be a dangerous nosocomial pathogen. A. baumannii has developed three basic properties to perfectly adapt to current healthcare settings: (i) Ability to colonize skin, mucous membranes, and devices and survive in the hospital environment; (ii) ability to express multiple virulence features; and (iii) extensive resistance to antimicrobial agents through enzymatic modification of antibiotics, target gene mutation, altered outer membrane permeability, and upregulated multidrug efflux pumps. Antibiotic policy should be formulated and updated according to the antibiogram results obtained in each hospital. Sensitive antibiotics as tigecycline and colistin should be used judiciously and within the antibiotic policy implemented. Primary caretakers should comply with the implemented antibiotic and infection control policies. This is not only done for the sake of protecting patients, but also for the preventing of the tremendous costs that arises intensely upon infection spread.

\section{References:}

1. Yeom J, Shin J-H, J-Y, Yang J-Y, Kim J, and Hwang G-S. "1H NMR-based metabolite profiling of planktonic and biofilm cells in Acinetobacter baumannii 1656-2," PLoS ONE, vol. 8, no. 3, Article ID e57730, 2013.View at: Publisher Site | Google Scholar.
2. Jeanette T, Ngan G, Balm M, et al. "Molecular characterization of NDM-1 producing Enterobacteriaceae isolates in Singapore hospitals." WPSAR 1 2012: 19.

3. Antunes LCS, Visca P, and Towner KJ. "Acinetobacter baumannii: evolution of a global pathogen," Pathogens and Disease 2014;71(3): 292-301, View at: Publisher Site $\mid$ Google Scholar.

4. Bergogne-Bérézin E. and Towner KJ. "Acinetobacter spp. as nosocomial pathogens: microbiological, clinical, and epidemiological features," Clinical Microbiology Reviews 1996; 9(2):148-165, View at: Publisher Site | Google Scholar.

5. Boucher HW, Talbot GH, Bradley JS. et al. "Bad bugs, no drugs: no ESKAPE! An update from the infectious diseases society of America," Clinical Infectious Diseases 2009; 48(1): 1-12, View at: Publisher Site | Google Scholar.

6. Clark NM, Zhanel GG, Lynch JP. Emergence of antimicrobial resistance among Acinetobacter pecies: A global threat. Curr. Opin. Crit. Care 2016;22: 491-499.doi:10.1097/MCC.0000000000000337. [PubMed] [CrossRef] [Google Scholar].

7. Peleg AY, Seifert H, Paterson DL. Acinetobacter baumannii: Emergence of a successful pathogen. Clin. Microbiol. Rev 2008;21:538-582. doi: 10.1128/ CMR.00058-07. [PMC free article] [PubMed] [CrossRef] [Google Scholar].

8. Bianco A, Quirino A, Giordano M, et al. Control of carbapenem-resistant Acinetobacter baumannii outbreak in an intensive care unit of a teaching hospital in Southern Italy. BMC Infect. Dis 2016;16:747. doi: 10.1186/s12879-016-2036-7. [PMC free article] [PubMed] [CrossRef] [Google Scholar].

9. Dijkshoorn L, Nemec A, Seifert H. An increasing threat in hospitals: Multidrug-resistant Acinetobacter baumannii. Nat. Rev. Microbiol 2007;5:939-951. doi: 10.1038/nrmicro1789. [PubMed] [CrossRef] [Google Scholar].

10. SmilineGirija AS, Priyadharsini JV. CLSI based antibiogram profile and the detection of MDR and XDR strains of Acinetobacter baumannii isolated from urine samples. Med. J. Islam. Repub. Iran 2019;33:3. [PMC free article] [PubMed] [Google Scholar]. 
11. Ghaith, Doaa Mohammad, Mai Mahmoud Zafer and Mohamed Hamed Al-Agamy, et al. "The emergence of a novel sequence type of MDR Acinetobacter baumannii from the intensive care unit of an Egyptian tertiary care hospital." Annals of Clin Micr and Antimicrobials 1. 2017:34.

12. Shanthi, Mariappan, Uma Sekar, Arunagiri Kamalanathan, and Balaraman Sekar. "Detection of New Delhi metallo beta lactamase-1 (NDM-1) carbapenemase in Pseudomonas aeruginosa in a single centre in southern India." T Indian J of Med Res 4. 2014: 546.

13. Mulani MS, Kamble EE, Kumkar SN, Tawre MS, Pardesi KR. Emerging Strategies to Combat ESKAPE Pathogens in the Era of Antimicrobial Resistance: A Review. Front Microbiol 2019;10:539. pmid: 30988669 View Article. PubMed/NCBI. Google Scholar.

14. Beigverdi R, Sattari-Maraji A, Emaneini M, Jabalameli F. Status of carbapenem-resistant Acinetobacter baumannii harboring carbapenemase: First systematic review and meta-analysis from Iran. Infect Genet Evol 2019;73:433-443. pmid:31176030 View Article. PubMed/NCBI. Google Scholar.

15. Takoi H, Fujita K, Hyodo H, Matsumoto M, Otani $\mathrm{S}$, Gorai $\mathrm{M}$, et al. Acinetobacter baumannii can be transferred from contaminated nitrile examination gloves to polypropylene plastic surfaces. Am J Infect Control 2019;0. https:// doi.org/10.1016/ j.ajic. 2019. 04.009. View Article. Google Scholar.

16. Gordon NC. and Wareham DW. "Multidrug-resistant Acinetobacter baumannii: mechanisms of virulence and resistance," International Journal of Antimicrobial Agents 2010; 35(3): 219-226, View at: Publisher Site | Google Scholar.
17. Kim YJ, Kim SI, Kim YR et al., "Carbapenemresistant Acinetobacter baumannii: diversity of resistant mechanisms and risk factors for infection," Epidemiology and Infection 2012; 140(1): 137-145, View at: Publisher Site | Google Scholar.

18. Poirel L. and Nordmann P. "Carbapenem resistance in Acinetobacter baumannii: mechanisms and epidemiology," Clinical Microbiology and Infection 2006; 12(9): 826-836, View at: Publisher Site | Google Scholar.

19. Jeon J, Lee J, Lee J. et al. "Structural basis for carbapenem-hydrolyzing mechanisms of carbapenemases conferring antibiotic resistance," International Journal of Molecular Sciences 2015; 16(12): 9654-9692, View at: Publisher Site | Google Scholar.

20. Pagano M, Martins AF, Barth AL. "Mobile genetic elements related to carbapenem resistance in Acinetobacter baumannii." Brazilian Journal of Microbiology 2016; 47(4):785-792, View at: Publisher Site | Google Scholar.

21. Khatun MN, Farzana R, Lopes BS, Shamsuzzaman SM. Molecular characterization and resistance profile of nosocomial Acinetobacter baumannii in intensive care unit of tertiary care hospital in Bangladesh. Bangladesh Med Res Counc Bull 2015; 41: 101-107.

22. CDC, Antibiotic Resistance Threats in the United States, CDC, Atlanta, GA, USA, 2013.

23. Papp-Wallace KM., Endimiani A., Taracila MA., and Bonomo RA. "Carbapenems: past, present, and future." Antimicrobial Agents and Chemotherapy 2011; 55(11): 4943-4960, View at: Publisher Site | Google Scholar. 